\title{
Statistical Estimation of Changes in the Dominant Frequencies of Structures in Long Noisy Series of Monitoring Data
}

\author{
Fanis Moschas ${ }^{1}$ and Eva Steirou ${ }^{2}$ \\ ${ }^{1}$ Laboratory of Geodesy and Geodetic Applications, Department of Civil Engineering, University of Patras, 26500 Patras, Greece \\ ${ }^{2}$ GFZ German Research Centre for Geosciences, Section 5.4 Hydrology, Telegrafenberg, 14473 Potsdam, Germany
}

Correspondence should be addressed to Fanis Moschas; fmoschas@upatras.gr

Received 24 September 2013; Accepted 12 November 2013

Academic Editor: Ting-Hua Yi

Copyright (C) 2013 F. Moschas and E. Steirou. This is an open access article distributed under the Creative Commons Attribution License, which permits unrestricted use, distribution, and reproduction in any medium, provided the original work is properly cited.

\begin{abstract}
Damage in structures is reflected in permanent changes of their natural frequencies and theoretically can be derived through measurements. Still, measurement-derived frequencies of structures usually reflect a superimposition of various effects, fluctuations due to environmental and loading conditions, noise, and possible permanent changes (damage or repair). The amplitude of the latter is usually of the same order of magnitude with the other effects; hence permanent shifts are masked by noise and cannot be identified, especially in long monitoring records. In order to overcome this problem, essential for the assessment of the structural health of various key structures, we adopt a statistical approach developed for the identification of shifts (inhomogeneities) in normally distributed climatological data, in particular the SNHT test. The efficiency of the SNHT was first tested on synthetic data and then on sets of estimates of dominant frequencies of a decaying pedestrian bridge. It was found that under certain conditions the SNHT can identify the location of shifts in dominant frequencies of structures; the amplitude of the shifts can then be easily computed. Since the efficiency of the test increases with the length of the time series, this test seems especially suitable for the analysis of long monitoring records.
\end{abstract}

\section{Introduction}

Structural damage in engineering structures is reflected in changes of their dominant frequencies, and there exist various techniques to infer damage from the analysis of measurements of the behaviour of the structures under various excitations and ambient conditions, usually accelerometer measurements [1-5]. However, in rare cases only observed changes in dominant frequencies are large (Figure 1(a)) and consistent with other compelling evidence (deterioration of the structure deduced from a visual inspection, highly reduced stiffness testified by increased deflections, correlation with a potentially destructive event, an earthquake, fire, etc.). In the majority of cases observed changes in dominant frequencies of structures are usually small (see Figure 1(b)) and of the same order of magnitude with transient changes, which may range up to $3-5 \%$ of the frequency value $[3,6,7]$.

In particular, measurement-based determination of dominant frequencies of structures may reflect a combination of several effects, including (1) measurement and computation bias, usually noise which lowers the accuracy of measurement-based results and calls for additional datafiltering $[8,9],(2)$ transient changes of the natural frequencies due to transient loading $[3,6,7]$ or environmental effects (rain, snow, etc.), occasionally effects with seasonal persistence, for example, icing of bridge-supporting cables, see $[10,11],(3)$ excitation of different, neighbouring modes, and (4) permanent changes (shifts) of the natural frequencies due to damage and gradual deterioration (or even repairs) of the structures $[1,3,5,6,12,13]$. For this reason, estimates of natural frequencies even before and after a certain potential damaging event (for instance an earthquake) tend to correspond to noisy data sets, that is, sets of fluctuating values, not giving clear evidence of a frequency shift.

In order to overcome this problem, a stochastic approach has been proposed in order to recognize whether two sets of data of measured frequency changes, before and after a critical event, indicate a real shift of frequencies and evidence 


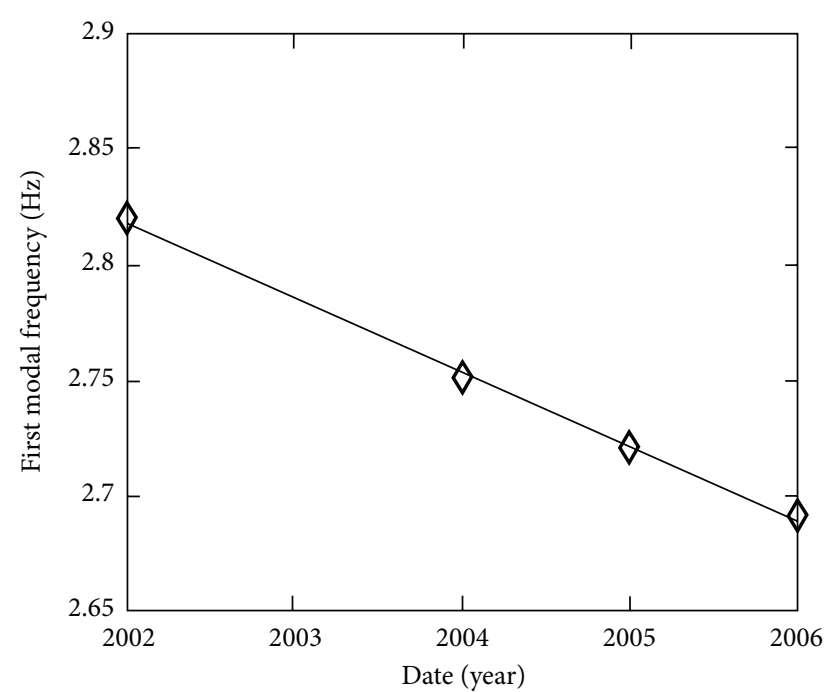

(a)

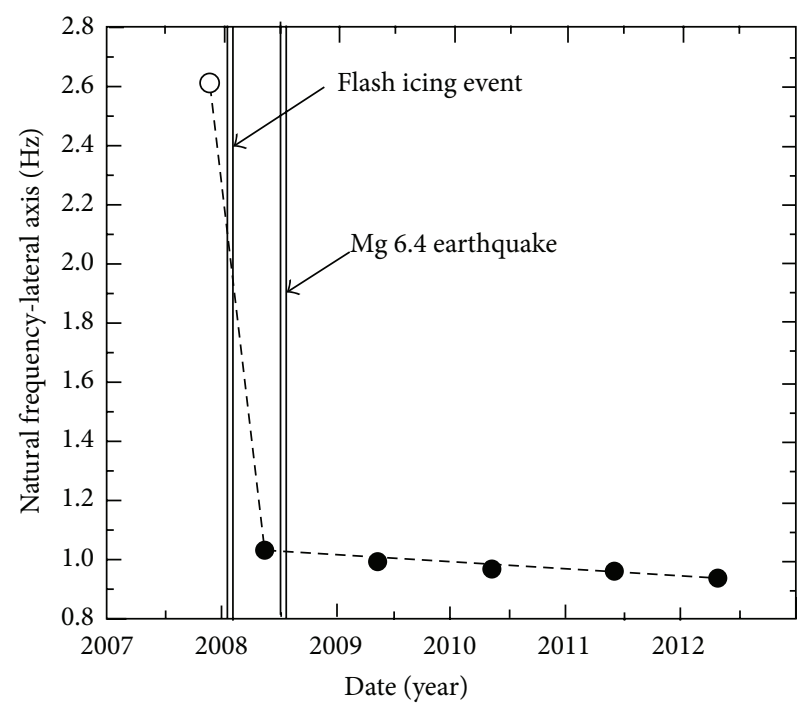

(b)

FIGURE 1: Examples of sudden and gradual drop of natural frequencies due to structural deterioration. (a) Natural frequencies of a concrete highway bridge derived from accelerometer measurements (modified after [3]) and (b) the extraordinary changes in the natural frequencies of the lateral axis of a timber pedestrian bridge derived from Robotic Total Station measurements (Stiros and Moschas, in review).

of damage (Moschas and Stiros, in review). This approach, based on a strict cross-correlation analysis, is especially suitable for cases of comparison of noisy spectral peaks corresponding to two different surveys and reflecting noisecontaminated data, mostly spectral leakage. However, this approach does not cover multi-epoch data, cases of a gradual decay (see Figure $1(\mathrm{~b})$ ), and so forth.

Another major problem is that permanent or periodic monitoring systems are currently available in several important structures (for instance, see [7]), leading to a large volume of data, including measurement-based estimations of dominant frequencies of the structure as a function of time. The time series of measurement-derived modal frequencies are likely to reflect a combination of some or of all the effects stated above; they are noisy and long, and even if there is some indication of drop of dominant frequencies, the available techniques do not permit to identify whether and where a shift can be documented and use this result for the evaluation of the structural health of the monitored structures. This makes it necessary to introduce more sophisticated stochastic approaches for the analysis of multi-epoch data.

This situation is somewhat similar to a problem that has been faced in the past in the analysis of climatological data, like sea level, rainfall, or temperature records. The latter are usually affected by unrecorded changes in the measuring procedure, in the sensors or their caging, and of the environment around the measuring stations, leading to shifts of the segments of the time series after the unrecorded change(s) and biased estimations of the climatic signature of the station records. The need to detect such undocumented changes (inhomogeneities, mostly shifts) in the available records led to the development of various inhomogeneity tests (shift detectors) based on different types of methodologies like linear regression analysis [14] or decomposition in orthogonal functions [15] among which is the Standard Normal Homogeneity Test (SNHT; see [16]). This test (and its variations) remains the simplest and one of the most popular tests for the detection of inhomogeneities in climatological data sets.

The use of the SNHT test is usually confined to climatological studies and has not been adopted in other fields of science and engineering. In this paper we use this test to detect small changes (statistically significant, permanent shifts) of frequencies of structures derived from multisurvey field measurements. This test is first applied on synthetic data describing hypothetical frequency measurements and shifts, and then it is used to analyze multi-epoch measurements of the dominant frequency of a timber bridge in Patras, Greece.

Application of this test is successful if the examined time series are dominated by white noise [17]. Clearly, no data to confirm such hypothesis exist for natural frequency estimates but for the present preliminary approach it can be assumed that the combination of the four effects mentioned above tends to satisfy this condition.

\section{Methodology}

2.1. Overview. In our study we use the SNHT on data-series of measurement-based estimates of the dominant frequencies of a certain bridge, in order to detect possible shifts in its 1st (fundamental) natural frequency. We assume that measured peaks express the same modal frequency (or the same set or nearby modal frequencies) and that the two sets differ a little from each other and such differences indicate noise, transient effects, and in some cases small permanent changes to be identified. These data can in a first approach be considered to be dominated by white noise.

We focus on estimates of dominant frequencies in spectra derived from accelerometer recordings, but this method is 
efficient for other types of measurements, such as frequencies derived from geodetic measurements of displacement, and so forth. Spectra are usually computed on the basis of FFT, but in our study we also use spectra based on the Least Squares Method [18] and the Normperiod Code [19]. A main advantage of this method is that it permits to estimate the level of statistical significance of the computed peaks and focus on the statistically significant peaks.

Then we apply the SNHT and try to identify at which point and at which level of significance a shift in frequencies can be documented. If the test is positive, the mean frequencies before and after the inhomogeneity are computed. The difference of the two values is a reliable estimate of the frequency change. Simple stochastic techniques permit to compute the variance of this estimate.

The SNHT is usually used for time series (e.g., monthly estimates of dominant frequencies), but under certain circumstances it can be used for sets of measurements corresponding to discrete epochs (surveys), each with several measurements (elements). In this last case, an equal number of frequency measurements should preferably be used for each survey. Additional limitations are imposed by the SNHT (see below). The overall process is summarized in the flowchart of Figure 2. Changes in the natural frequency of the studied bridge, due to gradual deterioration, were assumed to resemble abrupt shifts (inhomogeneities) between data sets from surveys carried out once per year. For this reason the simple SNHT, which is suitable for identifying a single shift in time series, was used for testing time series before and after a possible shift. As gradual deterioration of a structure may be expressed by multiple shifts of the natural frequency or by a gradual fall of the natural frequency during a certain period, different versions of the SNHT which permit to identify multiple shifts or changing trends [20] in the data set could be also used.

2.2. The Standard Normal Homogeneity Test (SNHT). The Standard Normal Homogeneity Test was introduced by Alexandersson [16] in order to identify inhomogeneities in time series of rainfall and temperature measurements, characterized by white noise. Inhomogeneities are defined as shifts in the mean value of the data before and after the inhomogeneity (shift). The same test and its variations are widely used in climatology [21].

The function of the test is to split a "suspect" time series in two segments and examine whether these two segments have different means. The process is repetitive, with the split point moving and covering all points of time series. For a point $v$ dividing a time series in two segments, a certain test variable $T_{v}$ is calculated for each pair of segments. A statistically significant inhomogeneity is identified if the value of $T_{v}$ corresponds to a peak exceeding a certain threshold value.

The main computational steps are the following.

(a) We examine a "suspect" time series $q_{i}$ with length equal to $n(i=1,2,3, \ldots n)$ with a possible shift at

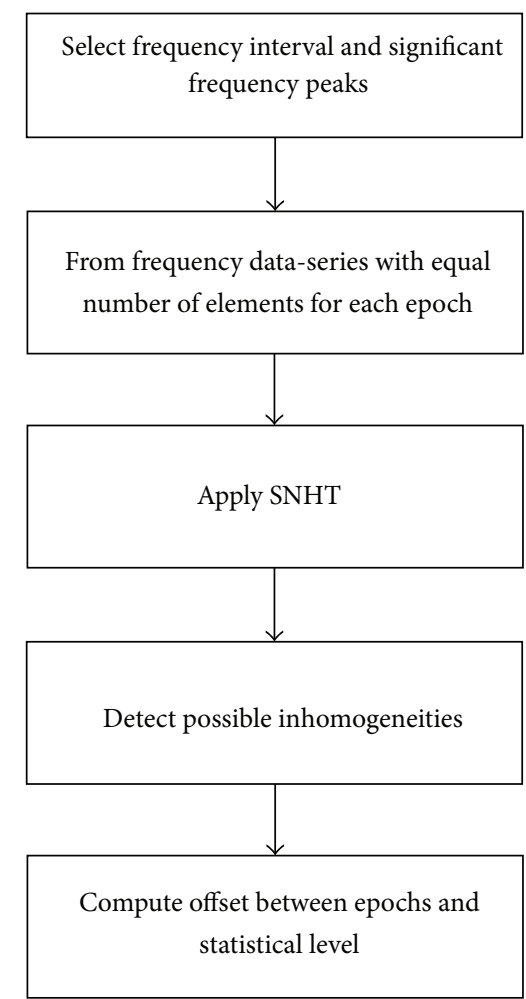

FIGURE 2: Flowchart summarizing the proposed methodology for detecting frequency shifts in spectra from structural vibration data sets using the SNHT.

point $v, 0 \leq v \leq n$ and we form a new normalized time series $z_{i}$ using

$$
z_{i}=\frac{\left(q_{i}-\bar{q}\right)}{s_{q}}
$$

with $\bar{q}$ equal to the mean value and $s_{q}$ to the standard deviation of $q_{i}$.

(b) Then we investigate whether all the elements $z_{i}$ follow a normal distribution $N(0,1)$ (null hypothesis $H_{0}$ ) or two different normal distributions $N\left(\mu_{1}, 1\right)$ and $N\left(\mu_{2}, 1\right)$ because of the shift (inhomogeneity, shift) at point $v$ (alternative hypothesis $H_{1}$ ). This is described by

$$
H_{0}: Z \in N(0,1), \quad \forall i
$$

$H_{1}:\left\{\right.$ for $1 \leq v \leq n, \mu_{1} \neq \mu_{2}$ we have

$$
\left.Z \in N\left(\mu_{1}, 1\right) \text { for } i \leq \nu Z \in N\left(\mu_{2}, 1\right) \text { for } i>v\right\} .
$$

(c) Hypotheses $H_{0}$ and $H_{1}$ are tested on the basis of a variable $T_{v}$ defined by

$$
T_{v}=v \bar{z}_{1}^{2}+(n-v) \bar{z}_{2}^{2}
$$

with $\left(\bar{z}_{1}\right)$ and $\left(\bar{z}_{2}\right)$ indicating the mean values of $z$ before and after a point $\nu$. This process is repeated for 
the various values of $v, 0<v<n$, and each time a variable $T_{v}$ is calculated. From the set of the various values of $T_{v}$, the time series of $T_{v}$ and the value defined by (4) is computed as follows:

$$
T_{0}=\max \left\{T_{\nu}\right\}
$$

If $T_{0}$ is larger than a critical value, an inhomogeneity at (or close to) point $\nu$ is documented. This critical value depends on the length of the compared time series and the chosen level of statistical significance and defines the statistical significance level of $T_{v}$. Details are given by Alexandersson and Moberg [20] and Sahin and Cigizoglu [21].

Practically, the estimation of a shift is reliable for relatively long time series (with $>5$ elements) and for shifts somewhat distant from the first and last point of the time series [16]. Calculations are made with codes freely available by various investigators. In this study we used the code presented by Steirou [17].

2.3. Amplitude of the Shifts. If a statistically significant shift is documented in the examined time series, the latter is split in two segments; a mean value is computed for each of them, and from their difference the amplitude of the shift is estimated. Its variance (or typical error) can be also easily computed.

\section{Detection of Shifts in Natural Frequencies Using the SNHT: Assessment Using Synthetic Data}

In order to test the limits of application of the SNHT in spectral data, we first applied the SNHT on synthetic data series simulating measurements of a modal frequency, in an accuracy-oriented test. At first we assume "initial," idealized time series presenting frequency estimates with no shifts or noise present. Then, in some of them, shifts were produced. Finally, white noise was added in these data series "dithering". This noise accounts for measurement errors, for multiple nearby modal frequencies, and for the apparent/transient shifts (see Section 1).

Similar evaluation tests have been carried out for synthetic climatological data characterized as white noise but also for more complex synthetic climatological data (mostly generated by an AR(1) model). Results are encouraging for the case of white noise or data with short-term memory but false-alarm problems are still faced in the case of data with long-term persistence [17].

The synthetic data-series used in the present study included frequency shifts from an initial, idealized natural frequency of $1 \mathrm{~Hz}$ with values equal to $0.00,0.02,0.04$, and $0.08 \mathrm{~Hz}$. The selection of the particular frequency does not limit the generality because SNHT analyses normalized data (1). White noise added to the data had a standard deviation $\sigma=0.04,0.06$, and $0.08 \mathrm{~Hz}$. The selected standard deviations correspond to a $4 \%, 6 \%$, and $8 \%$ deviation from the initial natural frequency of $1 \mathrm{~Hz}$. These values are compatible with the values of transient changes in natural frequencies of structures reported by various investigators $[3,6,7]$. The results from the application of the SNHT to the synthetic data are summarized in Figures 3-5.

From these figures, three main results were obtained.

First, shifts larger than the noise level $(\sigma)$ can be clearly identified by a peak in the $T_{v}$ value. Still, shifts statistically nonsignificant can be detected if their level is approximately $2 / 3$ of the noise level (data set 2, step $0.04 \mathrm{~Hz}$ in Figure 4).

Second, the location of the detected shift was close to the real location within a distance of maximum 3-4 point from the shift point (see dashed band in Figure 3) while on some cases the point of the shift, as identified by the SNHT, was on the same location with the real shift (see Figure 4).

Third, for the selected levels of data and uncertainties no false alarms (false identifications of frequency shifts) were detected.

These results encourage the application of SNHT in Structural Health Monitoring.

\section{Case Study: Detection of Natural Frequency Shifts of a Timber Pedestrian Bridge}

4.1. The Studied Bridge: Bearing System and Vibration Measurements. The tested timber footbridge is shown in Figure 6. It crosses the Kanellopoulos Avenue, at the northern entrance of Patras, Greece, and was constructed in 2000. The footbridge, with a maximum length of 29.5 and a central opening of $26.5 \mathrm{~m}$, consists of timber members and certain steel elements, all connected with metal bolts. The bridge deck is supported by two timber arches and two timber pylons (see Figure 6). Hence in side view the bridge can be regarded as a continuous beam based on two joint columns and with intermediate support provided by the timber arches. Overall, the structure is very stiff in the vertical axis. On the other hand, due to misconstruction or omission of certain crucial elements necessary to offer stiffness, the bridge is flexible in the lateral axis. Since its construction, significant loosening of the connections between the bridge members occurred and can be observed, while several steel members offering stiffness are permanently deformed. Furthermore, the bridge presents large lateral deflections of its deck which create a feeling of insecurity to the crossing pedestrians who have almost abandoned the bridge mainly after 2008. These changes are reflected in a dramatic, extraordinary shifting of its main lateral frequency deduced from analysis of measurements using a Robotic Total Station (RTS, or Robotic Theodolite, Figure 1(b)). For this reason it is usually avoided by pedestrians.

Concerning the vertical sense, the bridge is somewhat stiff, although there is a clear feeling to pedestrians of an episodic deterioration in 2008 and of a subsequent gradual deterioration.

Seven annual surveys aiming to measure the bridge response and evaluate the variations of its structural health with time were carried out between 2007 and 2013. The methodology adopted in all annual surveys was to excite various modes of the bridge deck using different types of excitation by a group of pedestrians (heel drops, walk, coordinate walk, running, coordinated stationary jumps, and jumps on the two sides of the deck-torsional excitation); see [8, 22-24]. 


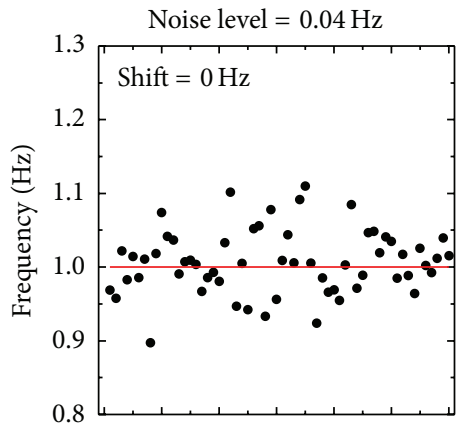

Noise level $=0.04 \mathrm{~Hz}$
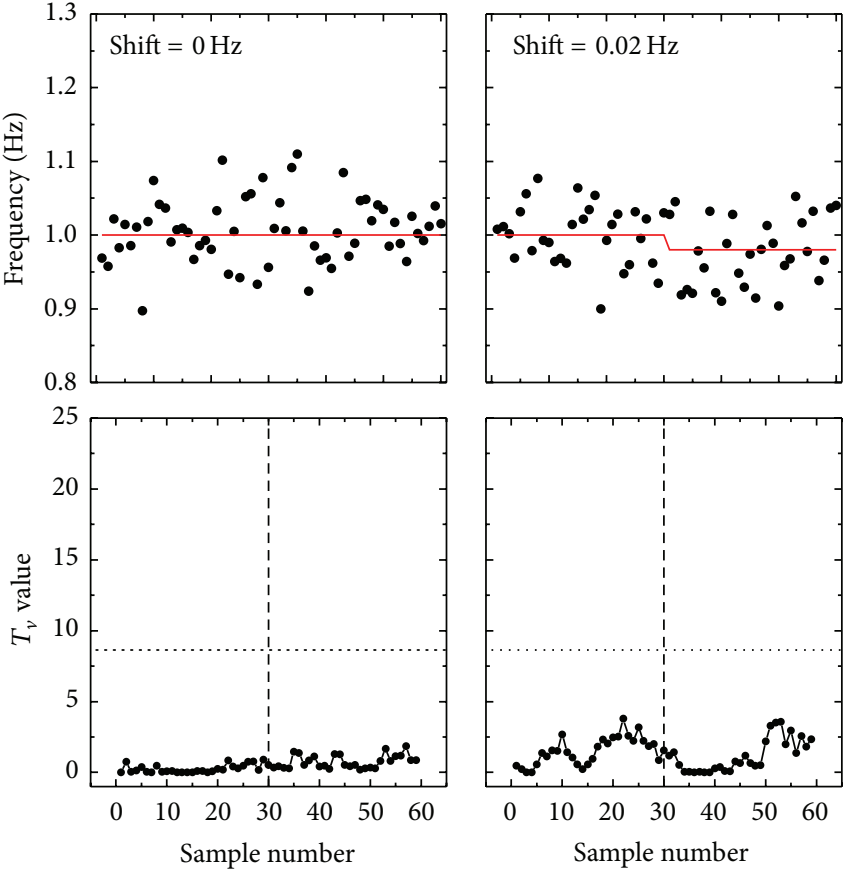

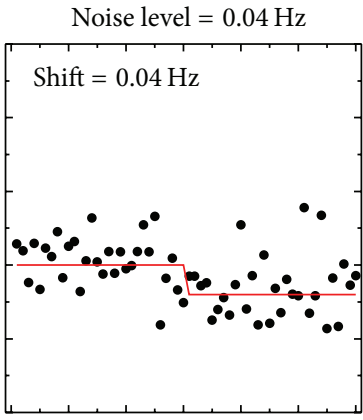

Noise level $=0.04 \mathrm{~Hz}$
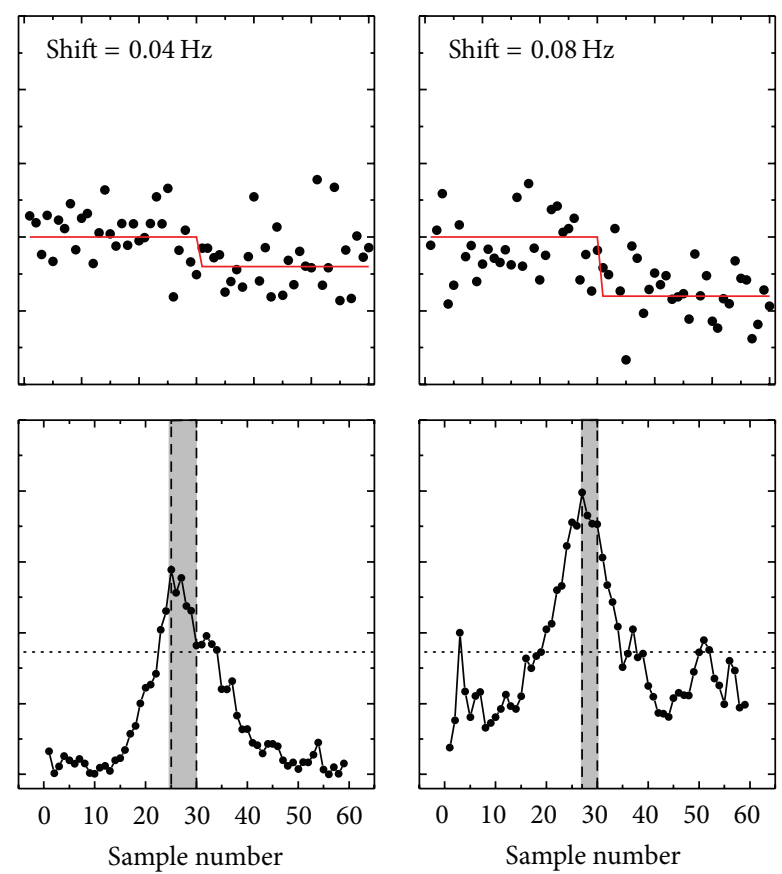

FIGURE 3: SNHT results for synthetic data set 1 with noise level $\sigma=0.04 \mathrm{~Hz}$ ( $4 \%$ of the frequency). Upper line: time series with no shift (shift $=$ $0 \mathrm{~Hz}$ ) and shifts equal to $0.02,0.04,0.08 \mathrm{~Hz}$, that is, lower and higher of the noise standard deviation. A red line indicates the frequency shift. Lower line: the test variable $T_{\gamma}$. A dotted horizontal line indicates the $95 \%$ level of statistical significance. Vertical dashed lines indicate the position of the real shift (always at point number 30) while a horizontal dashed line indicates the position of the maximum $T_{v}$. A gray area indicates the distance between the position of the maximum $T_{v}$ and the position of the real shift.
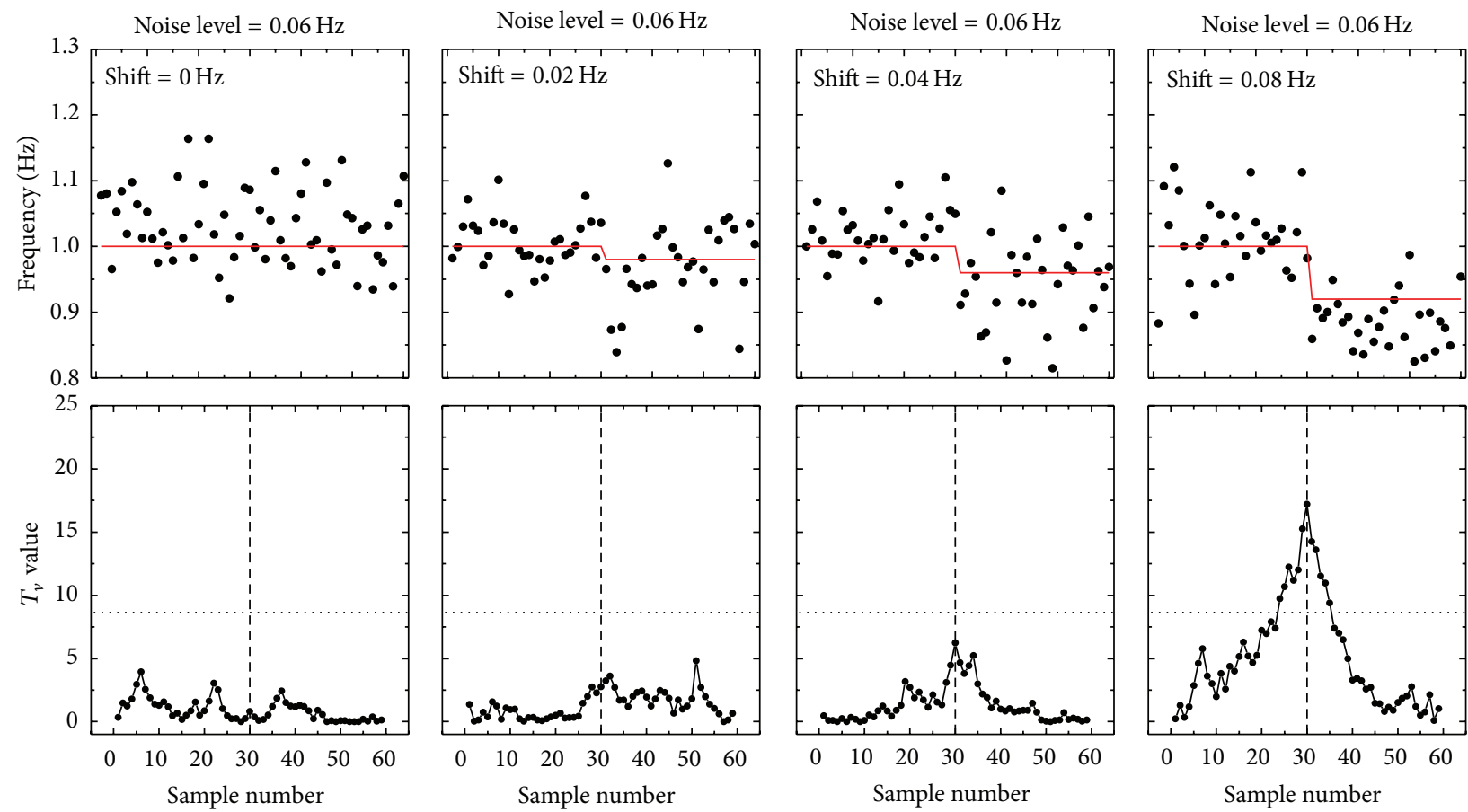

Figure 4: The same as Figure 2 for data set 2 with noise level $\sigma=0.06 \mathrm{~Hz}$ ( $6 \%$ of the frequency). 

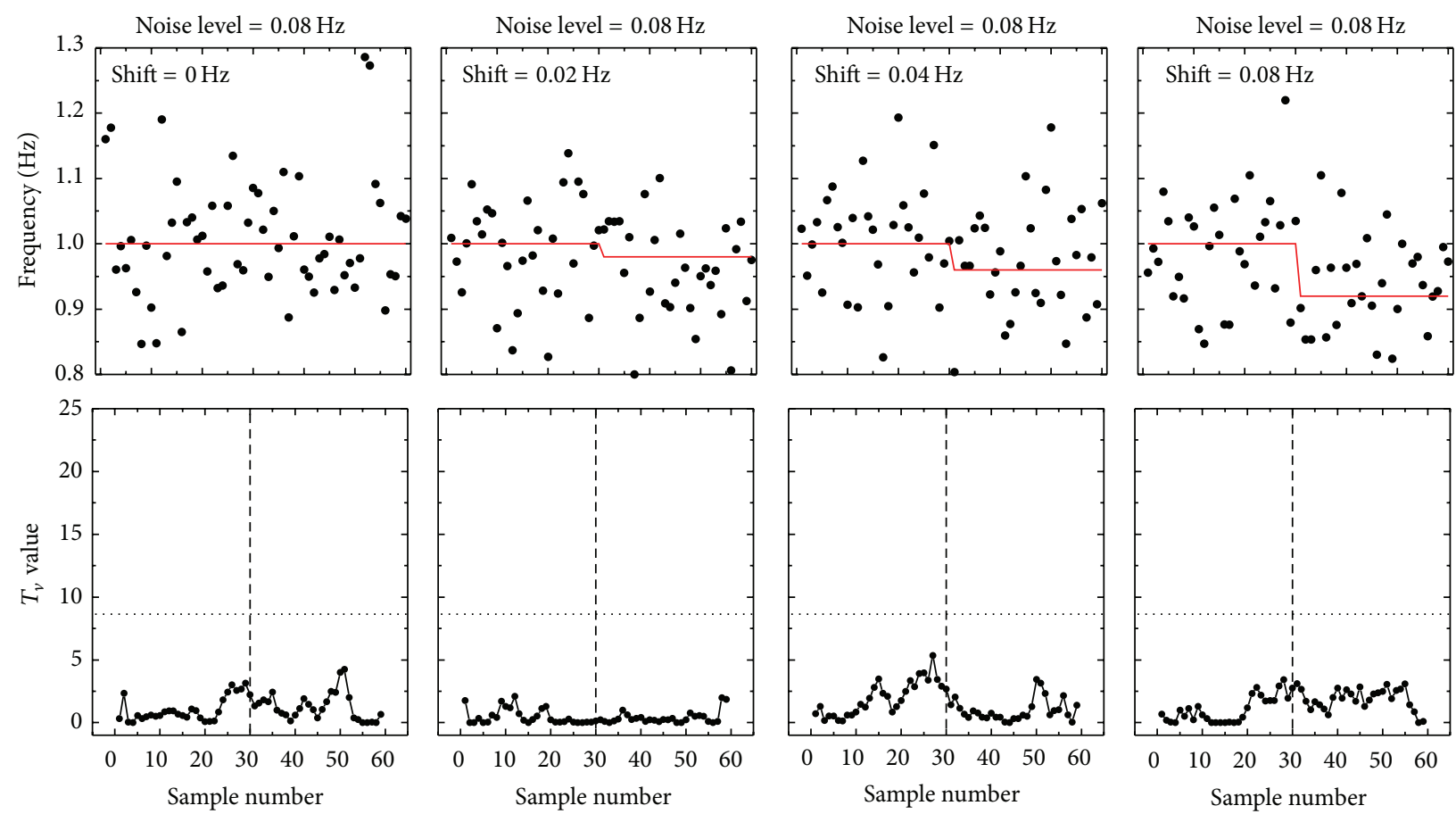

FIgURE 5: The same as Figure 2 for data set 3 with noise level $\sigma=0.08 \mathrm{~Hz}$ ( $8 \%$ of the frequency).

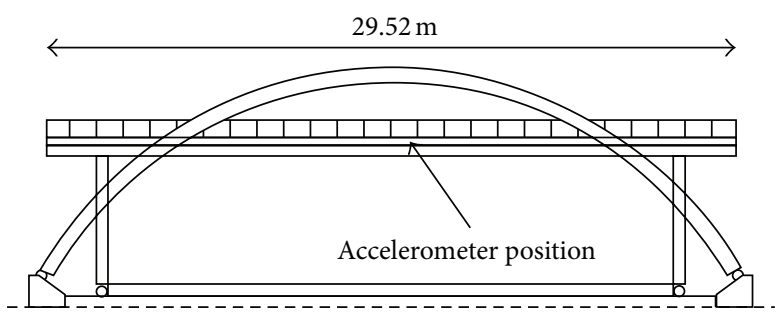

(a)

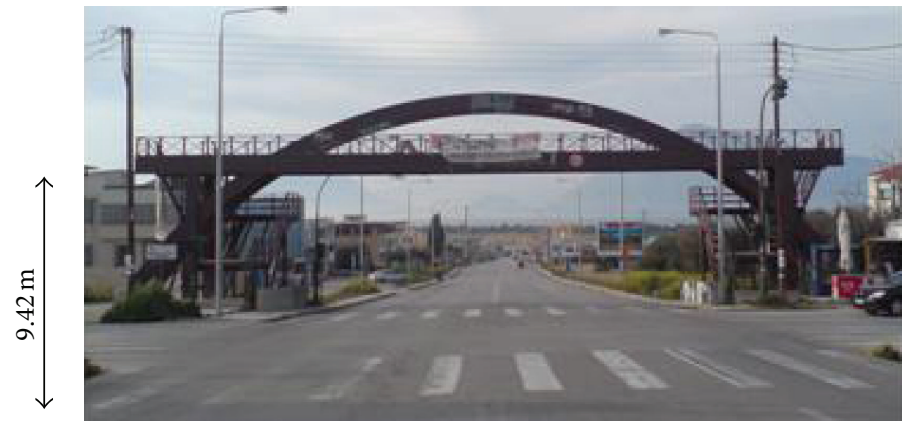

(b)

FIGURE 6: Structural concept (a) and side view (b) of the Kanellopoulos timber bridge in Patras, Greece. The bridge is relatively stiff along the vertical but flexible along the lateral axis, especially after episodic and gradual damage.

The bridge response was measured by several instruments including accelerometers, GPS, and Robotic Total Stations, occasionally by video recorders and a microwave interferometer (MA 200 Tellurometer).

This study is focused on measurements of the vertical response of the bridge using accelerometer records from the surveys of November 2007, May 2009, May 2010, May 2012, and April 2013. All surveys were carried out under nearly comparable loading and environmental conditions with the exception of 2012 when a larger number of pedestrians participated in the bridge excitation. Hence in this survey a higher dead load is expected to have led to slightly lower modal frequencies (see [6]).

During all surveys a set of Geo-Sig AC-23 acceleration sensor connected to a Geo-Sig GSR-24 recorder with GPS timing was used. In all surveys the accelerometer was stiffly connected to the bridge handrail with the exception of May 2009 when the sensor was connected to the floor of the bridge deck. In all surveys the accelerometers were installed at the mid-span of the bridge deck (see Figure 6).

\subsection{Spectral Analysis: Identification of the Bridge Natural} Frequencies. Spectra of displacements during the excitation events of each survey were computed using the Normperiod code (details on the Normperiod code are given in Section 2). Spectra corresponding to the lateral accelerations are presented in Figure 7 where spectra from different events are marked with a different color. A different number of events, ranging from 9 to 28 events, were obtained during each survey. From the results it is obvious that the main frequency peaks during 2007 were concentrated around $2.6 \mathrm{~Hz}$ while 

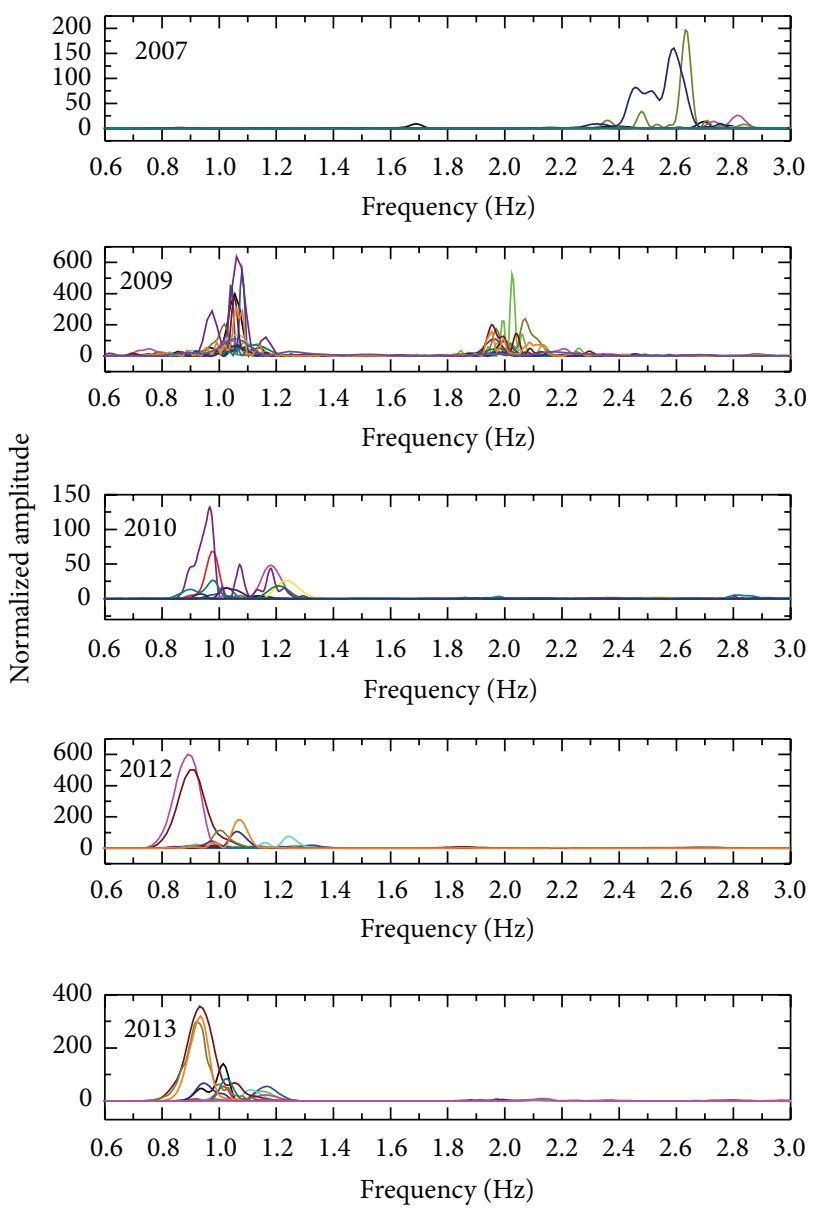

FIGURE 7: Spectra of the vertical response acceleration of the Kanellopoulos bridge during five annual surveys. Results were obtained after spectral analysis of the accelerometer recordings from different vibration events using the Normperiod code. Results from different events are marked with different colors. A dramatic shift of the dominating frequency peaks from 2.6 to $1 \mathrm{~Hz}$ can be identified between 2007 and 2009, followed by a gradual drop from 2009 to 2013. This shift is real and led to the abandonment of the bridge.

after 2009 the dominating frequency peaks are found around $1 \mathrm{~Hz}$ and $2.0 \mathrm{~Hz}$ and since 2010 at around $1 \mathrm{~Hz}$. Observed peaks are statistically significant. The overall tendency of shifting frequencies is common in all instruments used and consistent with the feelings of pedestrians, avoiding this bridge in the last years. Similar results were obtained when spectral analysis was carried out using FFT (see Figure 8).

Two significant effects can be identified from the spectra of Figures 7 and 8.

(a) A rapid fall of the first natural frequency in the lateral axis from 2.6 to $1 \mathrm{~Hz}$ between 2007 and 2009 .

(b) A gradual lowering of the first natural frequency in the lateral axis during the years 2009-2013.

The first effect indicates a major lowering of the stiffness of the bridge in the lateral axis that can only be attributed to loosening of the metal connections due to a rapid icing effect during the winter 2008, an extraordinary, regionally
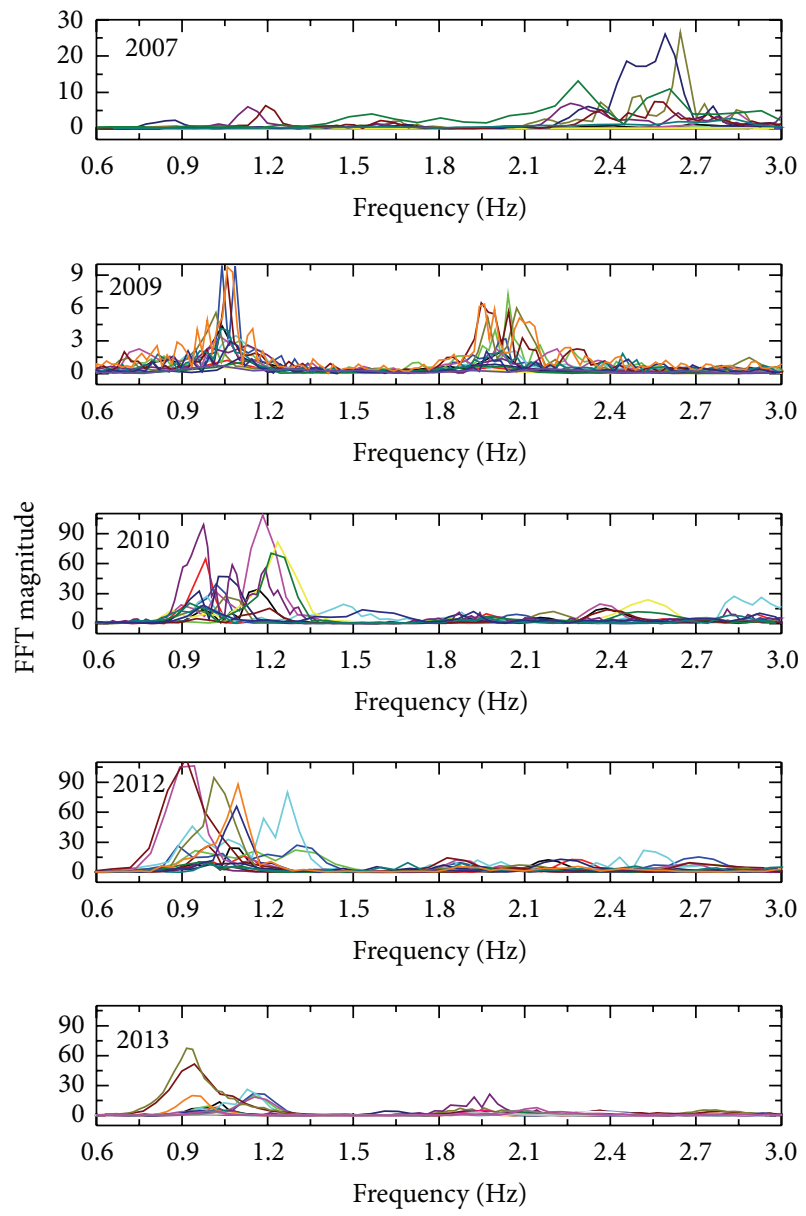

FIGURE 8: The same as Figure 7 but using FFT. Again different vibration events are marked with different colors.

catastrophic event. Another possibility is that the fall of the natural frequency is a measurement artifact, that the natural frequency close to $1 \mathrm{~Hz}$ was not excited during the first survey and the natural frequency close to $2.6 \mathrm{~Hz}$ was not excited during the last surveys (selective excitation). This is not, however, a likely explanation, for two reasons. First, because all surveys included different types of excitation, very similar in all surveys, and second, because a gradual loss of stiffness is observed in the vertical axis as well (see Moschas and Stiros (in review)), indicative of damage, obvious to pedestrians.

The shifting of the first natural frequency close to $1 \mathrm{~Hz}$ from 2009 to 2013 can be attributed to the gradual deterioration of the bridge bearing system, including the loosening of steel connections, permanent deformation of steel stiffening members, and decay of the timber members of the bridge that have been documented through the years.

From the spectra of Figure 7, the peaks above the 95\% level of statistical significance were isolated and plotted in Figure 9(a). From this figure a concentration of peaks around two main frequencies, one slightly lower and one slightly higher than $1 \mathrm{~Hz}$, can be identified. This distribution is identified after 2009 and may indicate two closely spaced natural frequencies. 


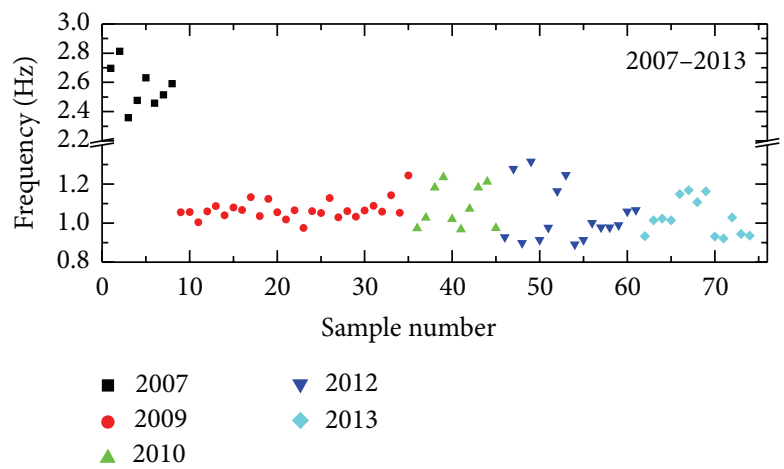

(a)

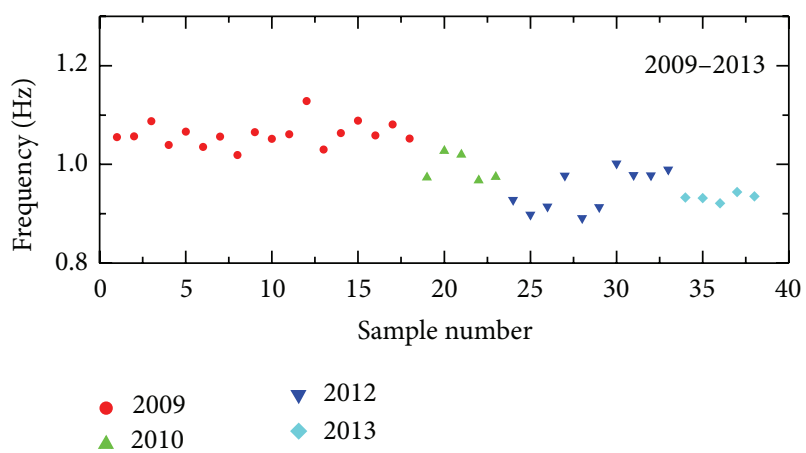

(b)

Figure 9: (a) All significant frequency peaks in the area between 0 and $3 \mathrm{~Hz}$ identified during the 5 annual surveys. A break is induced in the vertical axis for presentation reasons. A dramatic shift in frequencies is evident between 2007 and 2013. (b) Significant frequency peaks for the years after 2009 and further on. For the years 2010-2013 only the frequency peaks below $1 \mathrm{~Hz}$ were chosen and used in the analysis resulting in a smaller set of points compared with the set of (a). There is some evidence of lowering in the dominant frequency, but its location is not well defined.

The data set corresponding to the 2012 survey presents a pattern slightly different than that of the other years, with a larger separation between the two identified natural frequencies. Furthermore, the lower natural frequency lies at approximately $0.85 \mathrm{~Hz}$ and seems to increase towards $0.93 \mathrm{~Hz}$ in 2013. This difference can probably be attributed to the different loading conditions with a larger number of pedestrians (dead weight) during the 2012 survey. As discussed above results from the years 2010-2013 surveys contain two groups of significant frequency peaks: one lying slightly below and one slightly above $1 \mathrm{~Hz}$, probably indicating the existence of two natural frequencies. Since the present study is focused on detecting changes in the first natural frequency of the bridge deck only the peaks below $1 \mathrm{~Hz}$ were taken into consideration as they correspond to the lower, hence fundamental, natural frequency. The results from the 2009 survey along with the peaks below $1 \mathrm{~Hz}$ from the 2010, 2012, and 2013 surveys are plotted in Figure 9(b).

\subsection{Application of the SNHT on Natural Frequency Data} from Different Annual Surveys. The frequencies appearing in Figure 9(b) were used during the application of the SNHT for the detection of frequency shifts between the annual surveys.

The test was applied on the following data sets:

(a) on a data set covering years 2009-2013 (data of Figure 9(b)); the data of 2007 were ignored because the shift between 2007 and 2009 is obvious,

(b) on pairs of two yearly data sets from different years.

Application of the SNHT to the data set of years 20092013 (Figure 9(b)) led to the diagram of Figure 10. The test identified the dominant frequency drop between 2009 and 2010 , very close to the real boundary of the two surveys.

At a next step the SNHT was applied for combinations of data sets from different surveys (2007-2009, 2009-2010, 2010-2012, 2010-2013 and 2009-2013). For combinations of data sets covering more than two years (for example 20102013) the comparison was made only between the first and the
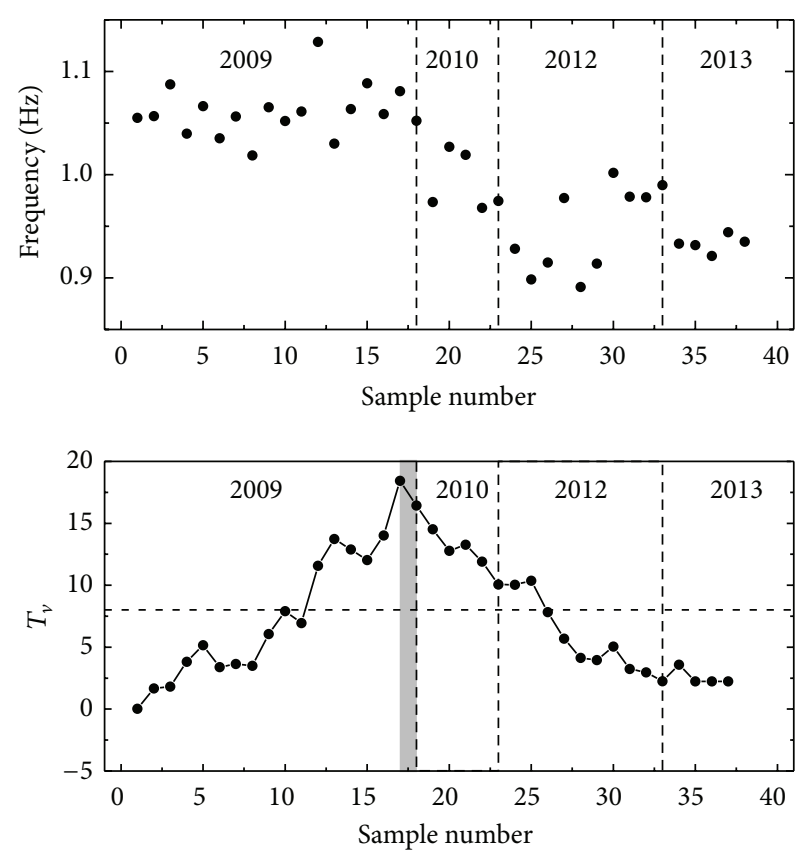

FIGURE 10: Results from application of the SNHT on the frequency estimates from the surveys covering the years 2009 to 2013. A clear frequency shift is detected between 2009 and 2010. A horizontal, dashed line in the bottom diagram indicates the $95 \%$ statistical significance level for the test, while vertical lines indicate the limits of the data sets corresponding to different annual surveys. A grayshaded area marks the distance between the point of the real shift and the point of the shift identified by the SNHT.

last survey ignoring intermediate surveys. No test between 2012 and 2013 was made for the reasons explained above.

The test was made this time not for the original available yearly data sets, of variable length (Figures 9 and 10), but for modified data sets of equal length produced by padding of each set (i.e., repeat of the original data) so that both sets in each pair contain 18 points. A main reason is that certain 

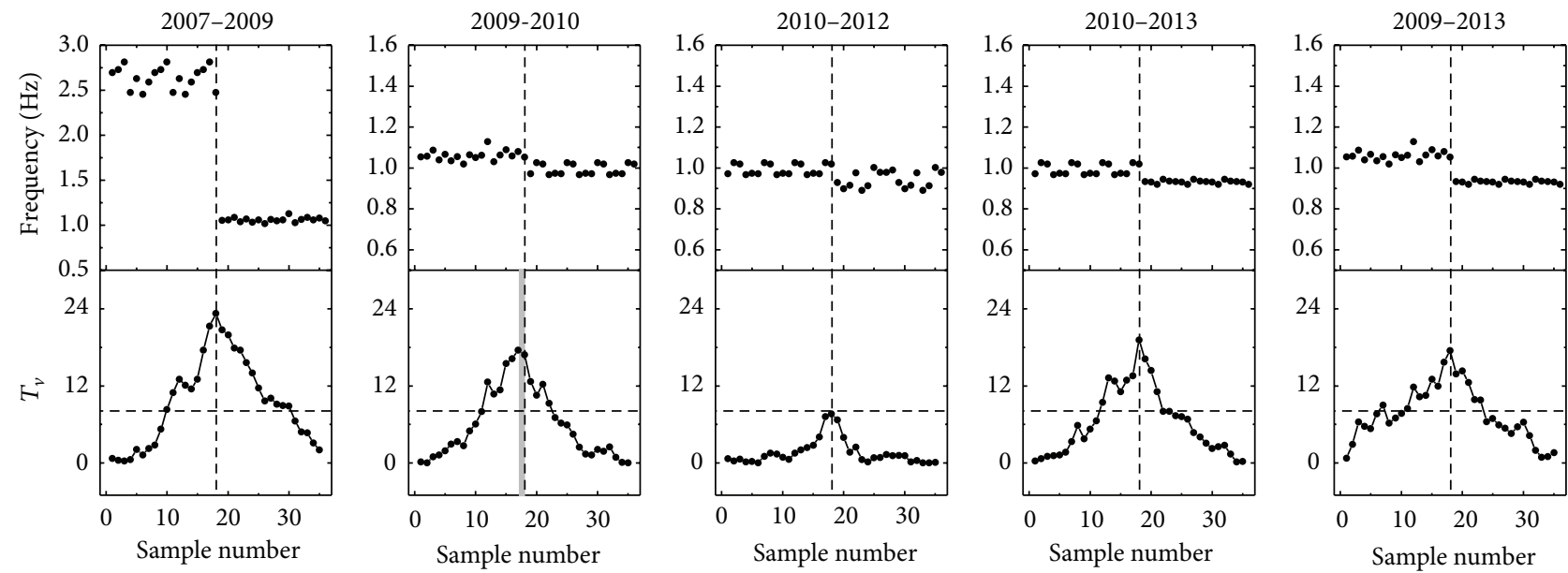

FIGURE 11: SNHT results for pairs of modified data sets from the annual surveys of the Kanellopoulos bridge. Top diagrams: time series of frequency peaks. Bottom diagrams: graph of the $T_{\gamma}$ function, with peaks indicating the location of possible shifts. A horizontal dashed line indicates the $95 \%$ level of statistical significance. The gray area in the diagram corresponding to the 2009-2010 test denotes the distance between the point of the shift identified by the SNHT and the point of the real shift.

surveys contain few data, while the test can identify shifts at some distance from the first and last point of the time series (see Section 2.2).

The results are shown in Figure 11. A frequency shift was identified at the boundary of the two surveys with the exception of the test between the 2009 and 2010 data sets.

\section{Discussion}

A large amount of monitoring data, spanning long period of time, can be collected from various bridges or other structures and can be exploited in order to estimate their dominant frequencies as a function of time. Frequency estimates are not idealized equal numbers, but fluctuating stochastic variables expressing measurement and computation errors, transient changes $[6,7,9,25]$ and damage-associated permanent changes $[4,26]$, as analysed in the Introduction. The amplitude of the permanent changes is usually of the order of the amplitude of the noise and of the transient changes usually up to a $3-5 \%$ value $[3,6,7]$ and only in extraordinary cases can be much larger (Figure 1(b)). For this reason, damage-associated changes in the dominant frequencies cannot be readily identified from the available time series of measurementderived dominant frequencies, that is, from long, apparently noisy data sets.

In order to solve this problem, we adopt a statistical approach developed for the identification of shifts in climatological, mostly temperature time series, especially the SNHT test. Various other tests, based on different types of methodologies, have been proposed in the past for the identification of inhomogeneities in data sets $[14,15]$. The SNHT is one of the simplest and most popular tests used for this task in the field of climatology. Other more complicated tests for identification of shifts in natural frequencies and structural damage have been proposed in the field of Structural Health Monitoring $[4,13,25]$. The SNHT was applied in sets of observations of dominant frequencies which were assumed to be dominated by white noise and suspect for a single small permanent shift.

At a first step the SNHT was applied on synthetic data simulating data sets with frequency shifts and added white noise with varying amplitude. The result of this assessment indicated that shifts with amplitude larger or equal to the standard deviation of the frequency estimates can be accurately detected by the SNHT, without false alarms, that is, identification of nonexisting shifts (see Figures 3-5).

At a next step the SNHT was applied on natural frequency data sets obtained from yearly repeated vibration measurements of a timber bridge in Patras Greece. A time series of statistically significant spectral peaks was formed from the spectral analysis of lateral displacements during events of forced excitation of the bridge (Figures 7 and 8). These data cover five surveys in 2007, 2009, 2010, 2012, and 2013. A major shift in the dominant lateral frequency is evident between 2007 and 2009, and for this reason the corresponding data sets were excluded from our analysis (Figure 9).

The analysis was made first for the series of yearly surveys between 2009 and 2013 and indicated a main shift between 2009 and 2010 (see Figure 10). Shifts between the other years were not detected, because of the structure of the particular test permitting to identify only single shifts in the time series. After a main shift is identified, the time series is split into two time series, and possible additional shifts are searched for each of them. When the test was applied between pairs of yearly data sets (Figure 11) a shift was identified between all surveys. The aim of the test is to identify statistically significant shifts in a time series. The results obtained are accurate, because the SNHT-identified shift is confirmed from independent evidence (measurement from various instruments, feeling of pedestrians, etc.).

SNHT is especially successful for long time series and for shifts at some distance from the first and last point of the time series [20]. Still the results previously presented are focusing on relatively small data sets; hence they indicate 
the lower level of tolerance of this test-finer and more accurate results are expected for longer time series. This makes SNHT especially suitable for long time series deriving from permanent monitoring systems, such as those which have been installed in various important structures (bridges, high-rise buildings, etc.) [7, 27]. Application of the SNHT on data sets noisier than the one used in the present study will be very interesting and will possibly broaden the limits of applicability of the test.

Our approach was limited to the identification of the shift. This is the real problem and its amplitude can be computed as difference of the mean values of the two segments of the time series defined by the shift (inhomogeneity).

Another limitation is that estimates of dominant frequencies were assumed to be dominated by white noise. This is certainly a simplification [28], because seasonal effects (e.g., icing of cables of suspension bridges $[10,11]$ ) may lead to data characterized by a different autocorrelation structure and transient effects may be misinterpreted as permanent shifts, especially in short time series. This can be avoided with a careful examination of the monitoring record, correlation of inferred shifts with meteorological effects, and so forth. In any case, any statistical test indicates only possible effects and should not be regarded as a black box.

Finally, in the present study frequency estimates were assumed to present abrupt shifts (inhomogeneities), due to the bridge deterioration through the years, between yearly surveys. For this reason the simple version of the SNHT which permits identification of single shifts in data sets was used. Different, more complicated versions of the SNHT permitting the identification of multiple shifts or of changes in the data set trend exist and could possibly be applied in data sets similar to the one studied in the present paper.

\section{Conclusion}

We have shown that a statistical test developed for climatological time series can be applied in time series of observed (better measurement derived) dominant frequencies of structures and hence detect possible damage (or ever repair or strengthening). The reason is that the amplitude of the permanent shifts is usually of the same order of magnitude with transient shifts and with measurement and computational noise, and hence it is difficult to identify permanent shifts without any specific statistical "tool." This test can be applied of course in other types of monitoring data, for instance, deflections.

\section{References}

[1] O. S. Salawu and C. Williams, "Bridge assessment using forcedvibration testing," Journal of Structural Engineering, vol. 121, no. 2, pp. 161-173, 1995.

[2] S. W. Doebling, C. R. Farrar, and M. B. Prime, "A summary review of vibration-based damage identification methods," Shock and Vibration Digest, vol. 30, no. 2, pp. 91-105, 1998.

[3] S. Soyoz and M. Q. Feng, "Long-term monitoring and identification of bridge structural parameters," Computer-Aided Civil and Infrastructure Engineering, vol. 24, no. 2, pp. 82-92, 2009.
[4] H. Li, T. Yi, M. Gu, and L. Huo, "Evaluation of earthquakeinduced structural damages by wavelet transform," Progress in Natural Science, vol. 19, no. 4, pp. 461-470, 2009.

[5] T.-H. Yi, H.-N. Li, and H.-M. Sun, "Multi-stage structural damage diagnosis method based on "energy-damage" theory," Smart Structures and Systems, vol. 12, no. 3, 2013.

[6] J. H. G. Macdonald and W. E. Daniell, "Variation of modal parameters of a cable-stayed bridge identified from ambient vibration measurements and FE modelling," Engineering Structures, vol. 27, no. 13, pp. 1916-1930, 2005.

[7] E. J. Cross, K. Y. Koo, J. M. W. Brownjohn, and K. Worden, "Long-term monitoring and data analysis of the Tamar Bridge," Mechanical Systems and Signal Processing, vol. 35, no. 1-2, pp. 16-34, 2013.

[8] F. Moschas and S. Stiros, "Measurement of the dynamic displacements and of the modal frequencies of a short-span pedestrian bridge using GPS and an accelerometer," Engineering Structures, vol. 33, no. 1, pp. 10-17, 2011.

[9] T.-H. Yi, H.-N. Li, and X.-Y. Zhao, "Noise smoothing for structural vibration test signals using an improved wavelet thresholding technique," Sensors, vol. 12, no. 12, pp. 11205-11220, 2012.

[10] C. T. Georgakis, J. H. Macdonald, and C. A. Taylor, "Non-linear analysis of wind-induced cable-deck interaction," in Proceedings of IABSE Conference, vol. 8, pp. 74-82, Cable-Supported Bridges-Challenging Technical Limits, Seoul, Korea, 2001.

[11] E. T. Ingólfsson, C. T. Georgakis, F. Ricciardelli, and J. Jönsson, "Experimental identification of pedestrian-induced lateral forces on footbridges," Journal of Sound and Vibration, vol. 330, no. 6, pp. 1265-1284, 2011.

[12] J. M. W. Brownjohn, P. Moyo, P. Omenzetter, and Y. Lu, "Assessment of highway bridge upgrading by dynamic testing and finite-element model updating," Journal of Bridge Engineering, vol. 8, no. 3, pp. 162-172, 2003.

[13] E. P. Carden and J. M. W. Brownjohn, "Fuzzy clustering of stability diagrams for vibration-based structural health monitoring," Computer-Aided Civil and Infrastructure Engineering, vol. 23, no. 5, pp. 360-372, 2008.

[14] L. A. Vincent, "A technique for the identification of inhomogeneities in Canadian temperature series," Journal of Climate, vol. 11, no. 5, pp. 1094-1104, 1998.

[15] M. Becker, M. Karpytchev, M. Davy, and K. Doekes, "Impact of a shift in mean on the sea level rise: application to the tide gauges in the Southern Netherlands," Continental Shelf Research, vol. 29, no. 4, pp. 741-749, 2009.

[16] H. Alexandersson, "A homogeneity test applied to precipitation data," Journal of Climatology, vol. 6, no. 6, pp. 661-675, 1986.

[17] E. Steirou, Investigation of Homogenization Errors in HydroClimatic Time Series with Long-Term Persistence, National Technical University of Athens, Athens, Greece, 2013, (Greek).

[18] N. R. Lomb, "Least-squares frequency analysis of unequally spaced data," Astrophysics and Space Science, vol. 39, no. 2, pp. 447-462, 1976.

[19] S. I. Pytharouli and S. C. Stiros, "Spectral analysis of unevenly spaced or discontinuous data using the "normperiod" code," Computers and Structures, vol. 86, no. 1-2, pp. 190-196, 2008.

[20] H. Alexandersson and A. Moberg, "Homogemzation of Swedish temperature data. Part I: homogeneity test for linear trends," International Journal of Climatology, vol. 17, no. 1, pp. 25-34, 1997. 
[21] S. Sahin and H. K. Cigizoglu, "Homogeneity analysis of Turkish meteorological data set," Hydrological Processes, vol. 24, no. 8, pp. 981-992, 2010.

[22] J. M. W. Brownjohn and A. Pavic, "Experimental methods for estimating modal mass in footbridges using human-induced dynamic excitation," Engineering Structures, vol. 29, no. 11, pp. 2833-2843, 2007.

[23] C. Gentile and N. Gallino, "Condition assessment and dynamic system identification of a historic suspension footbridge," Structural Control and Health Monitoring, vol. 15, no. 3, pp. 369-388, 2008.

[24] A. Rönnquist, E. Strømmen, and L. Wollebæk, "Dynamic properties from full scale recordings and FE-modelling of a slender footbridge with flexible connections," Structural Engineering International, vol. 18, no. 4, pp. 421-426, 2008.

[25] B. Peeters and G. De Roeck, "One-year monitoring of the Z24Bridge: environmental effects versus damage events," Earthquake Engineering and Structural Dynamics, vol. 30, pp. 149-171, 2001.

[26] O. S. Salawu, "Detection of structural damage through changes in frequency: a review," Engineering Structures, vol. 19, no. 9, pp. 718-723, 1997.

[27] K.-Y. Wong, "Instrumentation and health monitoring of cablesupported bridges," Structural Control and Health Monitoring, vol. 11, no. 2, pp. 91-124, 2004.

[28] D. Koutsoyiannis, “The Hurst phenomenon and fractional Gaussian noise made easy," Hydrological Sciences Journal, vol. 47, no. 4, pp. 573-595, 2002. 


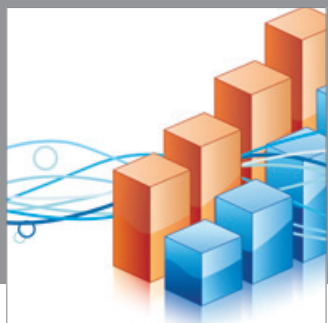

Advances in

Operations Research

mansans

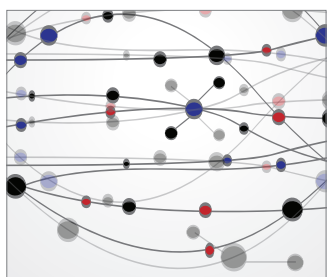

The Scientific World Journal
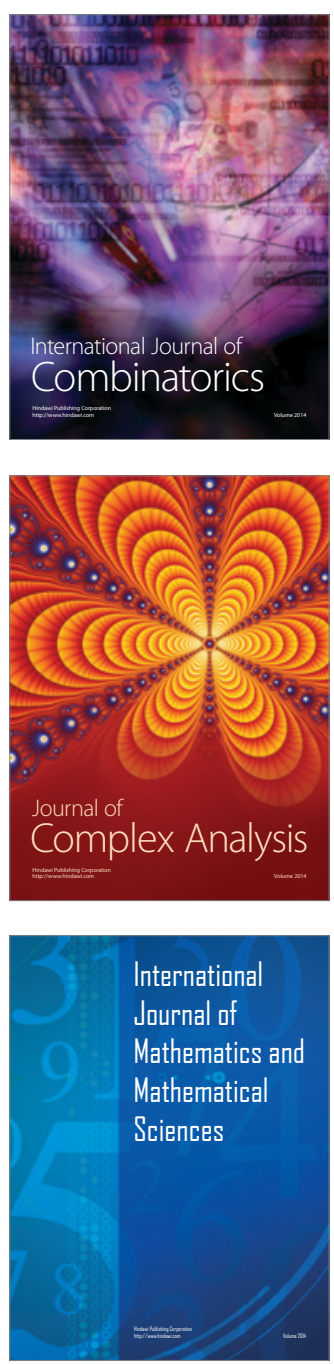
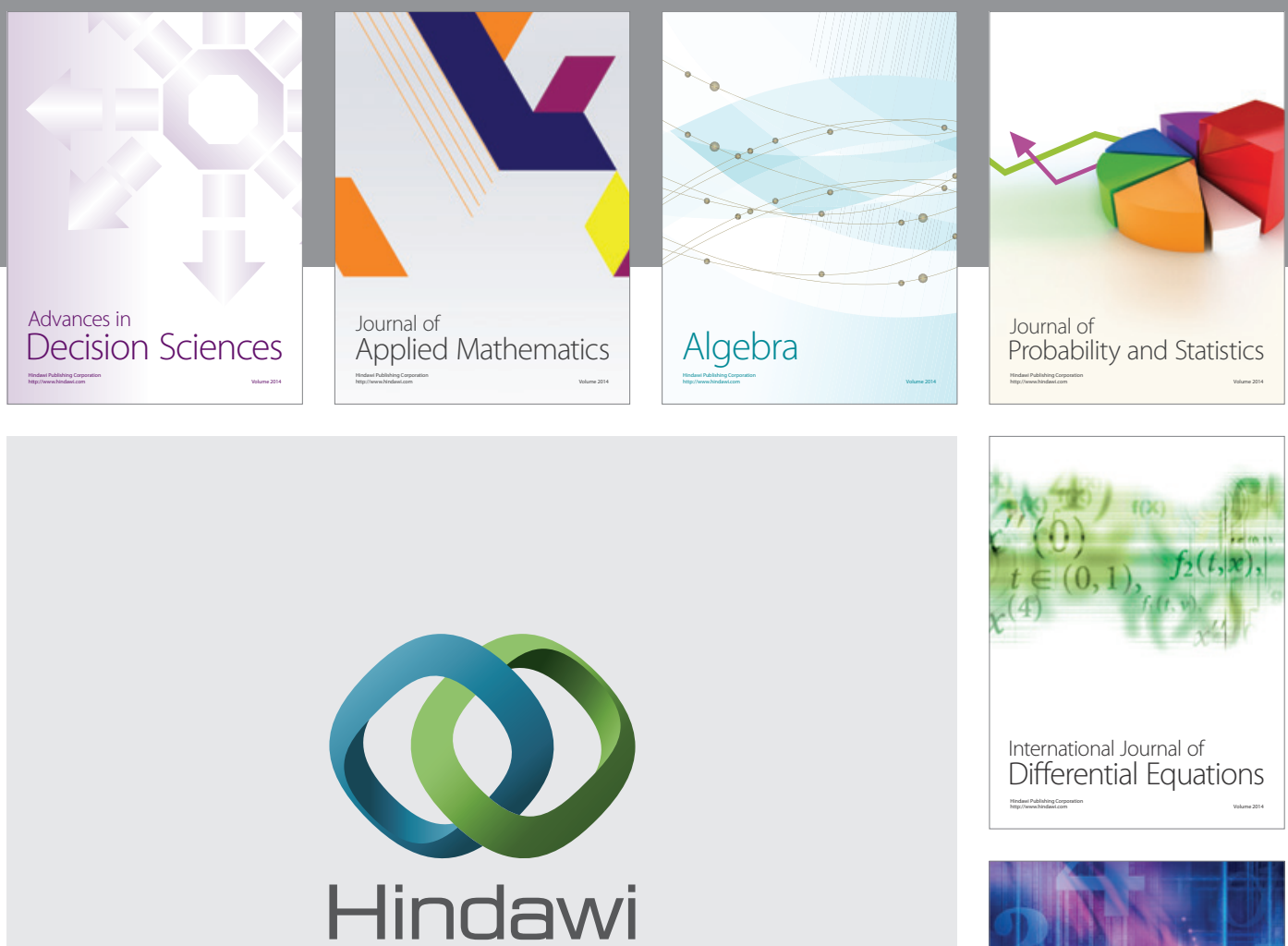

Submit your manuscripts at http://www.hindawi.com
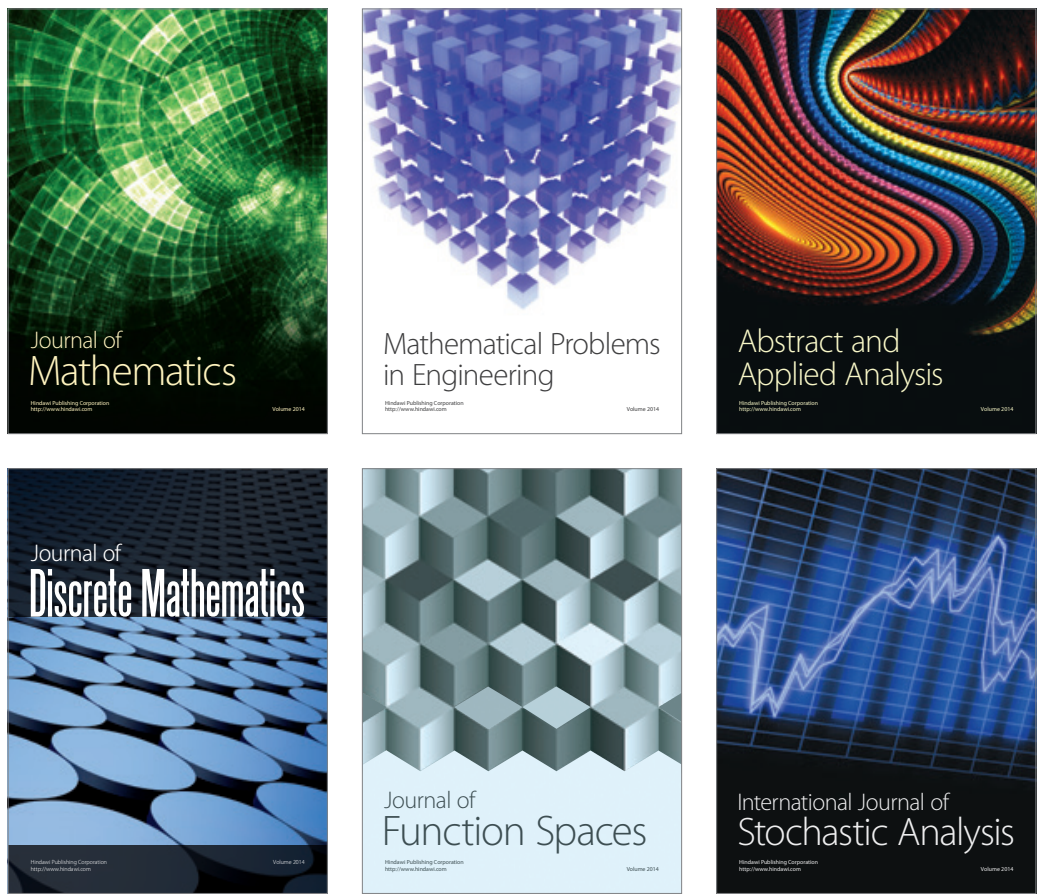

Journal of

Function Spaces

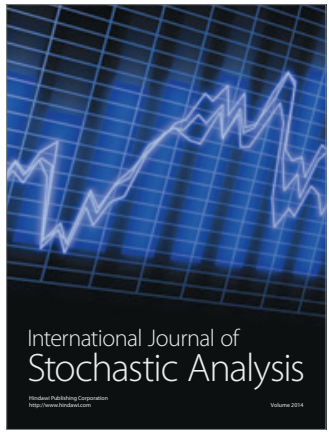

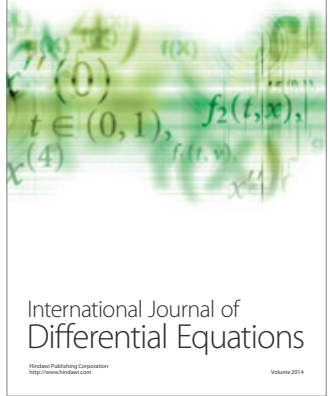
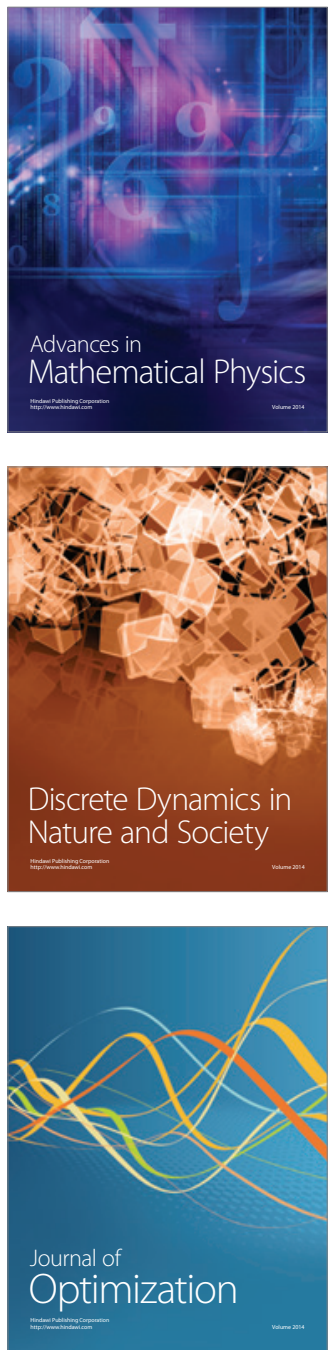